Psychother Psychosom 2020;89:117-118

DOI: $10.1159 / 000503570$

\section{Combined Internet-Based Cognitive-Behavioral and Chronobiological Intervention for Insomnia: A Randomized Controlled Trial}

\author{
Kim Dekker $^{\mathrm{a}}$ Jeroen S. Benjamins $^{\mathrm{b}, \mathrm{c}}$ Teodora Maksimovic ${ }^{\mathrm{a}}$ \\ Marco Filardi ${ }^{d}$ Winni F. Hofman ${ }^{\mathrm{e}}{ }^{\mathrm{f}}$ Annemieke van Straten ${ }^{\mathrm{g}}$ \\ Eus J.W. Van Someren ${ }^{\mathrm{a}, \mathrm{h}, \mathrm{i}}$

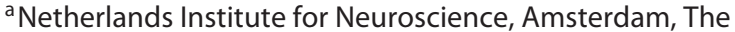 \\ Netherlands; ${ }^{b}$ Department of Social, Health and Organisational \\ Psychology, Utrecht University, Utrecht, The Netherlands; \\ 'Department of Experimental Psychology, Utrecht University, \\ Utrecht, The Netherlands; ${ }^{\mathrm{d}}$ Department of Biomedical and \\ Neuromotor Sciences - DIBINEM, University of Bologna, \\ Bologna, Italy; ${ }^{~}$ Brain and Cognition Group, Department \\ of Psychology, University of Amsterdam, Amsterdam, \\ The Netherlands; ${ }^{f}$ Personal Health Institute International, \\ Amsterdam, The Netherlands; ${ }^{9}$ Department of Clinical \\ Psychology, VU University Amsterdam and EMGO Institute \\ for Health Care and Research, Amsterdam, The Netherlands; \\ hDepartment of Psychiatry, Amsterdam University Medical \\ Center, VU University Amsterdam, Amsterdam, The Netherlands; \\ 'Department of Integrative Neurophysiology, Center for \\ Neurogenomics and Cognitive Research (CNCR), Amsterdam \\ Neuroscience, VU University Amsterdam, Amsterdam, The \\ Netherlands
}

\section{Dear Editor,}

Insomnia disorder is the second-most common mental health problem [1] and a major risk factor for depression [2]. Cognitive behavioral therapy for insomnia (CBTI) is the treatment of choice and has successfully been provided through the Internet (ICBTI) [3-6]. Unfortunately, the intervention is not sufficiently effective for all people that suffer from insomnia [7]. Meta-analysis also shows that it is not that common for ICBTI to improve sleep beyond the cutoff for normal sleep efficiency of 85\% [5], thus leaving ample room for additional improvement. It has moreover been stated that long-term maintenance therapies to reduce recurrence need to be developed [6].

A less studied type of intervention for insomnia, which might provide additional improvement if effective, is chronobiological treatment (CT). CT aims to enhance the entrainment and amplitude of the biological clock by utilizing its sensitivity to, and interaction with, regularly timed bright light, physical activity or body warming [8]. Although the European Sleep Research Society does recommend light and exercise in the treatment of insomnia disorder [3], support for their effectiveness is much smaller than for CBTI. The combination of CT and CBTI has been applied in one study, with promising results [9]. No prior study compared the effectiveness of CTs, ICBTI and their combination in treating insomnia disorder.

The primary aims of the present study were therefore to evaluate the initial and sustained separate, additive and interaction effects of CTs and ICBTI on sleep. To this end, we conducted a randomized controlled trial including 175 adults with insomnia who applied for ICBTI (see online supplement for details; for all online suppl. material see www.karger.com/doi/10.1159/000503570). After pre-assessment in week 0 (T0) randomization assigned half of them to receive 4 weeks of ICBTI in weeks $1-4$, the other half in weeks 6-9. In both groups, participants were then additionally randomized to receive scheduled CT, being either bright light, physical activity, warm baths, or a placebo (a deactivated ionizer) in weeks 1-4. A post-assessment was made during week 5 (T1) and a final follow-up assessment in week 10 (T2).

The preregistered primary outcome [10] was the change of diary-reported sleep efficiency (SE), a composite score that includes the three diagnostically defining complaints about sleep quality: difficulties initiating sleep, difficulties maintaining sleep, and early morning awakening. For each of the assessment weeks from T0 to T2, SE was calculated for each of the 7 days. Several secondary outcomes including expectation and compliance were assessed as well (online suppl. material).

Participants were diagnosed with insomnia disorder according to ICSD3 and DSM-5 [3] without another neuropsychiatric diagnosis. They did not use sleep medication daily nor performed shift work. The mean (SD) age was 51.0 (11.2) years (range 20-70), and $79 \%$ were female. Compliance to interventions and assessments was high and completed by 167 (95\%) of the participants at T1 and by $156(89 \%)$ at T2. The CONSORT flowchart (online suppl. material) provides detailed information on attrition, which did not differ between groups.

The overall SE at baseline was $69.4 \%$ (15.0\%). ICBTI increased SE by $6.69 \%$ (95\% CI: $0.34-13.03 \%, p=0.04)$ in addition to the increase between T0 and T1 seen across all participants irrespective of condition (5.55\%, 95\% CI: $0.95-10.15 \%, p=0.02)$. At T1, SE was not affected by any CT or CT by ICBTI interaction (all $p>$ 0.15 , online suppl. material). A noticeable effect of combining ICB$\mathrm{TI}$ with $\mathrm{CT}$ between $\mathrm{T} 0$ and $\mathrm{T} 1$ emerged only at $\mathrm{T} 2$. At $\mathrm{T} 2$ relative to T0, SE had increased by $12.89 \%$ (95\% CI: $10.06-15.71 \%$ ) in participants who combined ICBTI between T0 and T1 with any active $\mathrm{CT}$, which was almost twice ( $p=0.003$, online suppl. material) the increase seen in participants who had received ICBTI in combination with placebo CT (6.90\%, 95\% CI: 2.76-11.04\%) (Fig. 1). The effectiveness was robust, irrespective of the type of CT (scheduled light, activity, or baths).

Secondary outcomes indicated that adding CT to ICBTI resulted in significant late benefits at T2 as compared to not adding an active CT: sleep onset latency decreased by 23.60 versus 0.49 $\min (p=0.04)$; wake after sleep onset decreased by 64.29 versus

\section{KARGER}

karger@karger.com www.karger.com/pps
(C) 2019 The Author(s) Published by S. Karger AG, Basel

Karger Open access This article is licensed under the Creative Commons AttributionNonCommercial-NoDerivatives 4.0 International License (CC BYNC-ND) (http://www.karger.com/Services/OpenAccessLicense) Usage and distribution for commercial purposes as well as any distribution of modified material requires written permission.
Eus J.W. Van Someren

Department of Sleep and Cognition

Netherlands Institute for Neuroscience, Meibergdreef 47

NL-1105 BA Amsterdam (The Netherlands)

E-Mail e.j.w.someren@vu.nl 


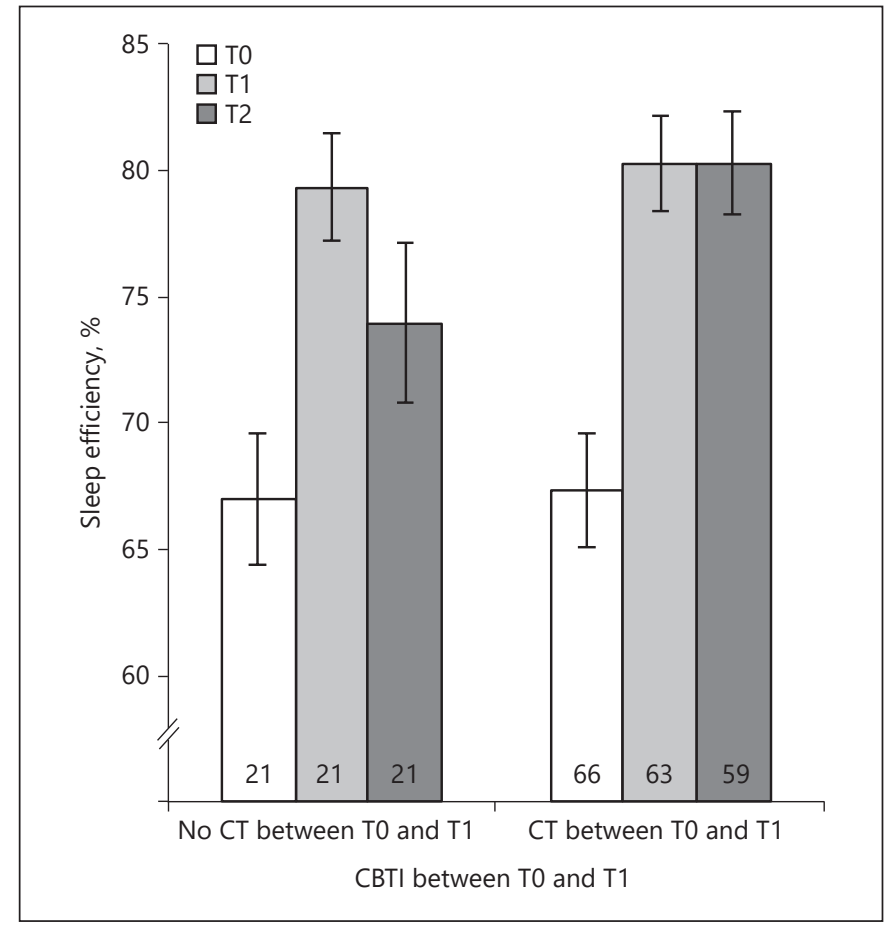

Fig. 1. Sleep efficiency at T0, T1, and T2 for participants who received ICBTI between T0 and T1, both without CT (left) and combined with CT (right). Bars show sleep efficiency at baseline (T0, white), week 5 (T1, light gray), and week 10 (T2, dark gray) for the groups that received ICBTI in weeks $1-4$ (between T0 and T1) either without CT ( 3 bars on the left-hand side) or with CT ( 3 bars on the right-hand side). Numbers in bars indicate the sample size of participants included in each condition at each time point. Light gray bars show little effect of adding CT on sleep efficiency immediately after CBTI. Comparison of the dark gray bars shows that valuable effects of adding CT emerge only at the follow-up: CT promotes maintenance of the initial sleep efficiency response to ICBTI.

$12.94 \mathrm{~min}(p=0.005)$; and total sleep time increased by 49.87 versus $17.38 \mathrm{~min}(p=0.01)$ (see online suppl. material for Figures, CI, details, and other outcomes).

Summarizing, this first study to evaluate within a single design the effects of CTs, ICBTI, and their interaction on sleep efficiency in insomnia disorder showed that stand-alone CT is not as effective as ICBTI. However, adding CT to ICBTI did bring on late benefits that appeared in the follow-up assessment in the sixth week after completing the interventions. Those that received ICBTI in combination with any active CT better maintained their initial gain in sleep efficiency by $6 \%$, fell asleep more easily by $23 \mathrm{~min}$, had less nocturnal wakefulness by $51 \mathrm{~min}$ and slept longer by $32 \mathrm{~min}$. The value of adding CT seems clinically relevant, notably because of the half hour of extra sleep it entails: meta-analysis indicates that sleep duration shows little improvement with stand-alone CBTI.

In conclusion, 4 weeks of ICBTI benefits the sleep efficiency of people suffering from insomnia disorder more than a similar period of stand-alone CT. A CT intervention for more than 4 weeks, or a combination of the $3 \mathrm{CTs}$, may be required for stronger immediate effects of stand-alone CT. However, the addition of a single CT to 4 weeks of ICBTI already gives clinically relevant delayed enhancements of the benefits of ICBTI.

\section{Disclosure Statement}

Author WHF developed the Somnio ICBTI, which is owned and exploited by Personal Health Institute International. The authors declare that they have no competing interests.

\section{Funding Sources}

This work was supported financially by the European Research Council ERC-ADG-2014-671084 INSOMNIA Grant and by the Perspective Programme OnTime, project 12188, of the Dutch Technology Foundation TTW, which is the applied science division of NWO and the Technology Programme of the Dutch Ministry of Economic Affairs.

\section{References}

1 Wittchen HU, Jacobi F, Rehm J, Gustavsson A, Svensson M, Jönsson B, et al. The size and burden of mental disorders and other disorders of the brain in Europe 2010. Eur Neuropsychopharmacol. 2011 Sep;21(9):65579.

2 Baglioni C, Battagliese G, Feige B, Spiegelhalder K, Nissen C, Voderholzer $U$, et al. Insomnia as a predictor of depression: a meta-analytic evaluation of longitudinal epidemiological studies. J Affect Disord. 2011 Dec; 135(1-3):10-9.

3 Riemann D, Baglioni C, Bassetti C, Bjorvatn B, Dolenc Groselj L, Ellis JG, et al. European guideline for the diagnosis and treatment of insomnia. J Sleep Res. 2017 Dec;26(6):675-700.

4 Zachariae R, Lyby MS, Ritterband LM, O’Toole MS. Efficacy of Internetdelivered cognitive-behavioral therapy for insomnia - a systematic review and meta-analysis of randomized controlled trials. Sleep Med Rev. 2016 Dec;30:1-10.

5 Seyffert M, Lagisetty P, Landgraf J, Chopra V, Pfeiffer PN, Conte ML, et al. Internet-delivered cognitive behavioral therapy to treat insomnia: A systematic review and meta-analysis. PLoSOne.2016Feb;11(2):e0149139.

6 Morin CM, Benca R. Chronic insomnia. Lancet. 2012 Mar;379(9821): 1129-41.

7 Harvey AG, Tang NK. Cognitive behaviour therapy for primary insomnia: can we rest yet? Sleep Med Rev. 2003 Jun;7(3):237-62.

8 Scheer FA, Pirovano C, Van Someren EJ, Buijs RM. Environmental light and suprachiasmatic nucleus interact in the regulation of body temperature. Neuroscience. 2005;132(2):465-77.

9 van der Werf YD, Altena E, van Dijk KD, Strijers RL, De Rijke W, Stam $\mathrm{CJ}$, et al. Is disturbed intracortical excitability a stable trait of chronic insomnia? A study using transcranial magnetic stimulation before and after multimodal sleep therapy. Biol Psychiatry. 2010 Nov;68(10):950-5.

10 Dekker K, Benjamins JS, Van Straten A, Hofman WF, Van Someren EJ. Effectiveness of internet-supported cognitive behavioral and chronobiological interventions and effect moderation by insomnia subtype: study protocol of a randomized controlled trial. Trials. 2015 Jul;16(1):292. 